\section{Probing the role of estrogen receptor isoforms in neonatal programming of neuroendocrine and behavioral functions}

\author{
Alexandre V. Patchev, 1 \\ Annemarie Wolff-Muscate, ${ }^{1,2}$ \\ Dieter Fischer,1 Osborne F.X. Almeida1 \\ 1 Max Planck Institute of Psychiatry; \\ 2Institute of Developmental Genetics, \\ Helmholtz Center, Munich, Germany
}

\section{Abstract}

Sex differences in the activity of the hypothalamus-pituitary-adrenal (HPA) axis in rats are programmed by neonatal estrogens; exposure of female neonates to estradiol $\left(\mathrm{E}_{2}\right)$ leads to overt defeminization of endocrine and behavioral functions in adulthood. $\mathrm{E}_{2}$ activates both estrogen receptor isoforms (ER $\alpha$ and ER $\beta$ ); these are widely expressed in the brain, and differentially regulate HPA axis activity in adulthood. However, the contributions of each ER isoform to the sex-specific organization of the neural mechanisms governing HPA axis function remain unknown. ER $\alpha, E R \beta$ agonists (PPT and DPN, respectively) or $\mathrm{E}_{2}$ were administered to female rats on days 1-10 of life. Animals subsequently underwent endocrine (HPA axis and reproductive) and behavioral profiling (anxiety-related and reproductive) in adulthood, and patterns of expression of relevant genes were monitored in limbic structures post mortem. Exposure of neonatal females to PPT or DPN led to distinctly different HPA secretory profiles, neither of which completely recapitulated the effects of $E_{2}$. Thus, whereas impaired glucocorticoid negative feedback was the most prominent effect of PPT treatment, increased basal corticosterone secretion was the most obvious characteristic of DPN-treated animals. Behavioral analysis revealed higher anxiety levels in PPT-treated animals, similar to those observed in $\mathrm{E}_{2}$-treated female neonates and control males; in contrast, DPN treatment was associated with reduced anxiety-like behavior. Parallel treatment-specific alterations in the expression of the genes encoding mineralocorticoid (MR) and glucocorticoid (GR) receptors in the hippocampus and amygdala and altered expression of ER $\beta$ mRNA in discrete brain regions, as well as disturbed ovarian activity, were also found; together, they suggest potential mechanisms that could account for the different endocrine and behavioral phenotypes observed.
Defeminization of HPA axis activity and associated anxiety-related behavior depends on balanced activation of $E R \alpha$ and $E R \beta$ during early postnatal life, rather than on the activation of a specific ER isoform. Long-term $\mathrm{E}_{2}$-, PPT- and DPN-induced alterations in the expression levels of GR and MR in the hippocampus and amygdala, as well as disrupted ovarian activity appear to be largely responsible for eliciting and maintaining the aberrant endocrine and behavioral phenotypes induced by estrogenization of neonatal females.

\section{Introduction}

Understanding the neurobiological basis of sex differences in the activity of the hypothalamus-pituitary-adrenal (HPA) axis is of medical relevance given the association between excessive glucocorticoid (GC) secretion and mood and anxiety disorders, conditions that occur more frequently in women. ${ }^{1}$ Women and female rodents secrete higher GC levels under both basal and stressful conditions; however, in contrast to males, healthy females show more efficient GC negative feedback regulation of adrenocorticotropin (ACTH) and glucocorticoid receptors (GR) in the pituitary, corticotrophin-releasing hormone (CRH), arginine vasopressin (AVP) and GR in the hypothalamus, and GR in the hippocampus. ${ }^{2-4}$ In adults of both sexes, these molecules are subject to dynamic regulation by gonadal steroids such as estradiol $\left(\mathrm{E}_{2}\right)$. In females, cyclical fluctuations in the secretion of sex steroids contribute to the regulation of glucocorticoid secretion $^{5-6}$ and a variety of behaviors in rodents $^{7}$ and primates, including humans; the influence of estradiol $\left(\mathrm{E}_{2}\right)$ on these functions is well known. ${ }^{8}$ On the other hand, neonatal exposure of female rats to $E_{2}$ results in the expression of a male-like HPA axis function. ${ }^{3}$ Since neonatal rats are considered to have a default female status, it is thought that the male phenotype results from the so-called organizing actions of neonatal estrogen, ${ }^{9}$ a view supported by the observation that neonatal castration of males prevents manifestation of masculine endocrine and behavioral features. ${ }^{10}$ Adult female gonadal secretions have been shown to affect HPA axis function through so called activational effects, whose magnitude and quality strongly depend on the organizing effects during early ontogeny. ${ }^{2}$

Estrogen actions are mediated by estrogen receptors (ER), of which there are two major isoforms (ER $\alpha$ and ER $\beta$ ). While ER $\alpha$ are predominantly expressed in brain nuclei implicated in the control of reproductive hormone secretion and behavior, ER $\beta$ are found in regions that are responsible for the regulation of non-reproductive functions, including HPA
Correspondence: Dr. Alexandre Patchev, Max Planck Institute of Psychiatry, Kraepelinstrasse 210, 80804 Munich, Germany.

Tel: +49.89 30622.397; Fax: +49.89.30622.461

E-mail: patchev@mpipsykl.mpg.de

Key words: HPA-axis, brain organization, ER isoforms, stress, anxiety.

Acknowledgements: the authors would like to thank Rainer Stoffel for excellent technical assistance.

Contributions: AVP, AWM and DF performed the experiments; AVP and OFX wrote the manuscript with critical input from all authors.

Funding: this work was funded by the European Union FP6 Integrated Project CRESCEND0. The funding agency did not influence the design of experiments and interpretation of the results and did not have any role in the preparation of this publication.

Conflict of interest: the authors report no conflicts of interest.

Received for publication: 24 August 2011. Revision received: 5 October 2011.

Accepted for publication: 15 October 2011.

This work is licensed under a Creative Commons Attribution NonCommercial 3.0 License (CC BYNC 3.0)

(c) Copyright A.V. Patchev et al., 2011

Licensee PAGEPress, Italy

Endocrinology Studies 2011; 1:e12

doi:10.4081/es.2011.e12

axis activity.11-12 Since $\mathrm{E}_{2}$ activates both $\mathrm{ER} \alpha$ and $\operatorname{ER} \beta$ in a relatively non-selective manner (EC50 values: $50 \mathrm{pM}$ and $200 \mathrm{pM}$ for $\mathrm{ER} \alpha$ and $\operatorname{ER} \beta$ respectively), ${ }^{13}$ questions regarding the individual contributions of each ER isoform to the organization of sex differences in HPA axis function remain open. This study addressed this issue by selectively activating ER $\alpha$ and ER $\beta$ with 4,4',4"'-(4-Propyl-[1H]-pyrazole1,3,5-triyl)trisphenol (PPT; EC50 of $200 \mathrm{pM}$ at $E R \alpha 410$-fold higher affinity for ER $\alpha$ than $\operatorname{ER} \beta)^{14}$ and 2,3-bis(4-Hydroxyphenyl)-propionitrile (diarylpropionitrile; DPN; $\mathrm{EC}_{50}$ of 0.85 $\mathrm{nM}$ at ER $\beta ; 170$-fold higher affinity for $\operatorname{ER} \beta$ than $\mathrm{ER} \alpha),{ }^{15}$ respectively. In addition to monitoring GC secretion under differing conditions, we also monitored a number of pathways involved in the regulation of the HPA axis and of anxiety-related behavior; the latter is influenced by adrenal and gonadal steroids. ${ }^{16-17}$ Our results highlight the importance of co-activation of both ER isoforms during sex-specific organization of the brain since activation of just one isoform results in erroneous programming of both neuroendocrine and behavioral 
functions. These findings are interesting from an environmental health perspective, as many environmental pollutants and endocrine disrupting compounds show differential affinities for the two ER isoforms. ${ }^{18-19}$ Further, our experiments draw attention to the fact that the disruptive effects of neonatal estrogenization paradigms on ovarian secretions and their receptive targets must be considered when interpreting the results from such experiments. Specifically, our results hint that sex differences of HPA axis function arise from impairment of activational estrogenic effects due to impairment of sex-steroid secretion and ER expression patterns in the brain, which are the consequence of the neonatal sex-steroid milieu.

\section{Materials and Methods}

\section{Animals and treatment paradigms}

All experiments were conducted in compliance with the Code of Ethics of The Endocrine Society and European Union Directive on Animal Experiments (Directive 2010/63/EU); specific procedures were approved by the ethics committee of the Government of Upper Bavaria, Germany (Permit 2531-22-07). Timed pregnant Wistar rats were purchased from Charles River Laboratories (Sulzfeld, Germany) on gestation day 15 and were housed individually under standard conditions (lights on: 18.00, lights off: 6.00). On the day of birth, litters were culled (8-10 pups), with equal distribution of males and females across litters. On postnatal days 1-14 (PND 1-14), litters were assigned to one of four treatment groups: vehicle (peanut oil), estradiol benzoate (EB, $7.5 \mu \mathrm{g} ; \mathrm{n}=13$ ), 4,4',4"-(4-Propyl[1H]-pyrazole-1,3,5-triyl)trisphenol (PPT, ER $\alpha$ agonist, $50 \mu \mathrm{g} ; \mathrm{n}=10$ ), or 2,3-bis(4Hydroxyphenyl)-propionitrile (DPN, ER $\beta$ agonist, $50 \mu \mathrm{g} ; \mathrm{n}=14$ ). Estradiol benzoate (Sigma Aldrich, Deisenhofen, Germany), PPT and DPN (both from Tocris, Bristol, UK) were initially dissolved in absolute ethanol and peanut oil (final ethanol: $0.001 \%$ ) and injected subcutaneously in a volume of $0.1 \mathrm{~mL}$ on every second day. Choice of doses was based on the relative binding affinities of PPT and DPN to ER $\alpha$ and ER $\beta$ respectively ${ }^{14-15}$ and the relative transcriptional efficacies of these compounds compared to previously used compounds. ${ }^{20}$ Upon weaning on PND 21, animals were ear-marked and housed in groups of 4 under an inversed light rhythm. Ovarian cyclicity was monitored (vaginal smear cytology) from post natal days 80 to 121 and female sexual behavior was assessed between days 114 to 121 . Animals were tested for locomotor and anxiety-related behaviors in the open field 21 and elevated plus maze ${ }^{22}$ start- ing at ca. 130 days of age, with an interval of at least 1 week between each test. All methods of behavioral analysis are described below. Blood samples (tail vein) were collected for evaluation of HPA axis activity and glucocorticoid negative feedback (PND 130); serum was stored at $-20^{\circ} \mathrm{C}$ until assayed for hormones. Animals were killed on PND 150.

\section{Assessment of female sexual behavior}

Female sexual behavior was evaluated according to established protocols. ${ }^{20}$ In brief, vasectomized, sexually experienced male Wistar rats were placed in the testing cage and allowed to habituate for 5 min before being presented with estrous females. The number of mounts, lordosis responses and ejaculations were used to compute the lordosis quotient over an observation period of $5 \mathrm{~min}$.

\section{Assessment of anxiety-related behavior}

Thigmotaxis was evaluated (5 min) in an open field arena (LxBxH: $70 \times 70 \times 50 \mathrm{~cm}$; nonreflecting white $\mathrm{PVC}$ ) according to established protocols. ${ }^{21}$ Randomly-cycling non-treated females were used as controls (to ensure a normal distribution of the phases of the oestrous cycle throughout the experiment) and both tests were performed under 100 lux illumination. Central and peripheral line crossings as well as time spent in the central area of the arena were scored. Anxiety-related behavior was evaluated in the elevated plus maze test ${ }^{22}$ (LxBxH: $50 \times 10 \times 40 \mathrm{~cm}$, with openarm edges $0.5 \mathrm{~cm}$ high; placed $70 \mathrm{~cm}$ above the floor). The number of entries into, and the time spent in the open compartments of the maze were evaluated over a period of $5 \mathrm{~min}$. Events in the open field apparatus and elevated plus maze were video-recorded and subsequently scored by an investigator blind to the treatments.

\section{Characterization of HPA axis activity}

Basal and stress-induced corticosterone secretion was monitored in serial blood samples as reported elsewher.23 Serial blood samples (ca. $20 \mu \mathrm{L}$ ) were obtained while animals were in their home cages over approximately $20 \mathrm{~s}$.

Samples for estimation of diurnal fluctuations in corticosterone were collected at the circadian zenith (06:00) and nadir (18:00). Immediately thereafter, animals were exposed to an emotional stressor for $2 \mathrm{~min}$; to this end, animals were placed in an empty cage and exposed to an air puff delivered with a hair dryer. Blood samples were obtained 30 and 180 min later, to determine maximal corticosterone responses and shut-off of the endocrine response to stress. After a resting period of 3 days, animals were subjected to a dexamethasone suppression test (DST). For this, animals were given a bolus intraperitoneal (i.p.) injection of dexamethasone (Fortecortin ${ }^{\circledR}$, Merck, Darmstadt, Germany; $10 \mu \mathrm{g} / \mathrm{kg}$ BW in a volume of $0.2 \mathrm{~mL}$ ) at 24:00. Animals were blood sampled at 06:00 (the expected time of the circadian peak of corticosterone secretion). Serum samples were stored at $-20^{\circ} \mathrm{C}$ until hormone assay.

\section{Tissue processing}

Animals were sacrificed at the circadian zenith of HPA axis activity (06:00) by rapid decapitation. Brains were rapidly removed from the skull, snap-frozen in pre-chilled isopentane and kept at $-80^{\circ} \mathrm{C}$ until further processing. Six serial coronal $(10 \mu \mathrm{m})$ cryosections were prepared from the PVN (bregma 1.53 to -1.78 ) amygdala (bregma -1.78 to -2.0 ) and dorsal hippocampus (bregma -2.45 to 4.60) and micropunches from the remaining parts of these areas of interest were obtained as previously described. ${ }^{24}$ Sections and micropunches were stored at $-80^{\circ} \mathrm{C}$ until further processing.

\section{RNA isolation and qPCR}

RNA was isolated from micro-dissected brain areas using RNAeasy ${ }^{\circledR}$ kits (Qiagen, Hilden, Germany), and 100 ng RNA were used for cDNA synthesis (RevertAid ${ }^{\circledR}$ kit; Fermentas, St. Leon-Rot, Germany). Quantitative polymerase chain reaction (qPCR) was performed using SYBR Green I Master mix on a LightCycler 480 (Roche Applied Science, Mannheim, Germany). Expression levels of mRNAs of interest were normalized against levels of Mas mRNA since preliminary studies showed that Mas per se is not regulated by sex or hormonal status (data not shown). Primer sequences are listed in Table 1 (5'-3').

\section{GR and MR mRNA expression in hippocampus and amygdala}

Labeled ribonucleotide probes for the detection of GR and MR protein-encoding transcripts were produced from linearized plasmids using in vitro transcription kits with $\mathrm{T} 7$, T3 and Sp6 RNA polymerases (Promega, Madison, WI) and [ $\left.{ }^{35} \mathrm{~S}\right]$-dUTP (Perkin Elmer, Rodgau, Germany). The GR and MR expression plasmids were a generous gift from Dr. J. L. Arriza.25-26 Cryosections were permeabilized and hybridized according to published protocols. ${ }^{27}$ Autoradiograms (BioMax MR; Kodak, Rochester, NY) were analyzed by densitometry on two sections per animal, using the NIH software Scion Image Beta 4.2.0. Individual averaged transmittance levels were converted to specific radioactivity by third-order polynomial equations generated from co-exposed $14 \mathrm{C}$ 
standards (ARC, St. Louis, MO, USA).

\section{Hormone measurements}

Serum corticosterone levels in serial blood samples, and estradiol, progesterone and luteinizing hormone (LH) concentrations in probes derived from trunk blood, were determined using commercially available radioimmunoassay (corticosterone: DRG Instruments, Marburg, Germany) or enzyme immunoassay (estradiol and progesterone: Beckman Coulter, Krefeld, Germany; LH: Millipore, Schwalbach, Germany) kits.

\section{Statistics}

Data are presented as either group means \pm SEM or scatter plots with medians. Group means were compared by either parametric or non-parametric 1-way ANOVA and appropriate post-hoc tests (Tukey-Kramer or KruskalWallis, respectively). The threshold of significance was defined as $\mathrm{P}<0.05$.

\section{Results}

\section{Programming versus disorganiza- tion of HPA axis activity}

Several aspects of HPA axis function differ markedly in the two sexes. For example, previous studies showed that females secrete higher levels of corticosterone under both baseline and stressful conditions. ${ }^{2-3}$ Further, those studies demonstrated that these endocrine profiles are subject to defeminization by neonatal exposure of female rats to estradiol benzoate (EB). ${ }^{3,4}$ As shown in Figure 1, those earlier findings with EB were reproduced in the present work, in which neonatal estrogenization resulted in attenuated corticosterone secretory responses to stress (Figure $1 \mathrm{~A}, \mathrm{P}<0.0001$, $\mathrm{F}=26.4$ ) as well as reduced night time (zenith) baseline levels of corticosterone (Figure 1B, $\mathrm{P}<0.0001, \mathrm{~F}=45.5)$. Together, these results attest to the ability of neonatal EB to program the neuroendocrine system to elicit phenotypically male HPA axis responses in rats with a female genotype.

Since EB non-selectively activates both ER $\alpha$ and $\operatorname{ER} \beta$, HPA axis activity in adulthood was next assessed in females that had been exposed to selective agonists of either $\mathrm{ER} \alpha$ (PPT) or ER $\beta$ (DPN) during neonatal life. As compared to normal female rats, PPT-treated animals had significantly lower daytime baseline corticosterone levels $(\mathrm{P}<0.01, \mathrm{~F}=62.2)$ although night time levels did not differ between the two groups (Figure 1B). Notably, neither PPT nor DPN treatments reproduced the effects of EB (daytime corticosterone: DPN > EB > PPT; night-time corticosterone: DPN > PPT > EB; Figure 1B). Interestingly, vehicle-,

Table 1. Primer sequences (5'-3') used for quantitative polymerase chain reaction.

\begin{tabular}{lll} 
Gene & Forward primer sequence & Reverse primer sequence \\
Mas & AGTACCGTCGTGGCTGCTGAGAA & GGCGTTGTCCATGGCGGTCAT \\
SCC & TGCAGCTGCCTGGGATGTGATT & ATGGAGGTCGTGTCCACCCCTCC \\
\hline$S t A R$ & GGCGTCGGAGCTCTCTACTTGGTTC & ACCTTGCCCACACCTGGCAC \\
$3 \beta-H S D$ & TTGTGGTGAGTCAGAAGACCAGGG & TGCTCCTGTCACCAGGCAGC \\
\hline$A C T H R$ & TTGTGACCATGCGACGCACC & CATGGTGATGCCGCTCCCTGT \\
$E R \alpha$ & GGCTGCGCAAGTGTTACGAA & CATTTCGGCCTTCCAAGTCAT \\
\hline$E R \alpha$ & TTCCCGGCAGCACCAGTAACC & TCCCTCTTTGCGTTTGGACTA \\
$G R$ & ACCTCGATGACCAAATGACC & AGCAAAGCAGAGCAGGTTTC \\
\hline$M R$ & CGTACAAACATACGAACAGCCA & TAGAACCTCTGCCAACTCTGTC \\
\hline
\end{tabular}

Mas, NM 053825; SCC, steroid side-chain cleavage; StAR, Steroid acute regulatory protein; 3 -HSD, 3 $\beta$-hydroxysteroid dehydrogenase; ACTHR, Adrenocorticotropin (ACTH) receptor; ER $\alpha$, Estrogen receptor $\alpha$; ER $\beta$, Estrogen receptor $\beta$; GR, glucocorticoid receptor; MR, mineralocorticoid receptor.

PPT- and DPN-treated rats all responded to an acute stressor with significant increases in corticosterone secretion. However, the between-group magnitudes of response differed remarkably (approximately 5-fold, 8-fold and 2 -fold in the vehicle-, PPT- and DPN-treated groups, respectively; Figure 1A). In terms of relative magnitude of response to stress, the DPN-treated animals showed closest resemblance to the EB-treated group; however, it should be noted that the DPN group displayed high basal corticosterone levels, and that the attenuated corticosterone response to stress in DPN-treated rats is unlikely to be the result of reduced steroidogenic capacity since the levels of the mRNAs encoding for critical regulators of adrenocortical steroidogenesis (StAR protein, P450scc, 3 $\beta$-hydroxysteroid dehydrogenase, and the ACTH receptor) were unchanged (data not shown). Considered together with the results obtained with $\mathrm{EB}$, it is concluded that whereas dual occupation of ER during neonatal life programs the female HPA axis to express a male-like phenotype, selective activation of either ER $\alpha$ or $E R \beta$ results in malprogramming of the central mechanisms that regulate HPA axis function.

\section{Disruption of central mechanisms regulating HPA axis function}

Homeostatic control of corticosterone secretion is maintained through a series of regulatory loops that are sensitive to the negative feedback actions of corticosterone. The dexamethasone suppression test (DST) serves as a powerful tool to assess the efficacy of corticosteroid negative feedback at both brain and pituitary levels. ${ }^{28}$ Here, administration of the DST revealed that, as compared to vehicle- EBand DPN-treated females, PPT-treated females display impaired corticosteroid-mediated negative feedback (Figure $2 \mathrm{~A}, \mathrm{P}<0.0001, \mathrm{~F}=10.1$ ). Since males are known to be less sensitive to glucocorticoid negative feedback in the DST,28 it is interesting to note that neonatal PPT treatment of females resulted in even greater insensitivity to dexamethasone $(\mathrm{P}<0.001)$.
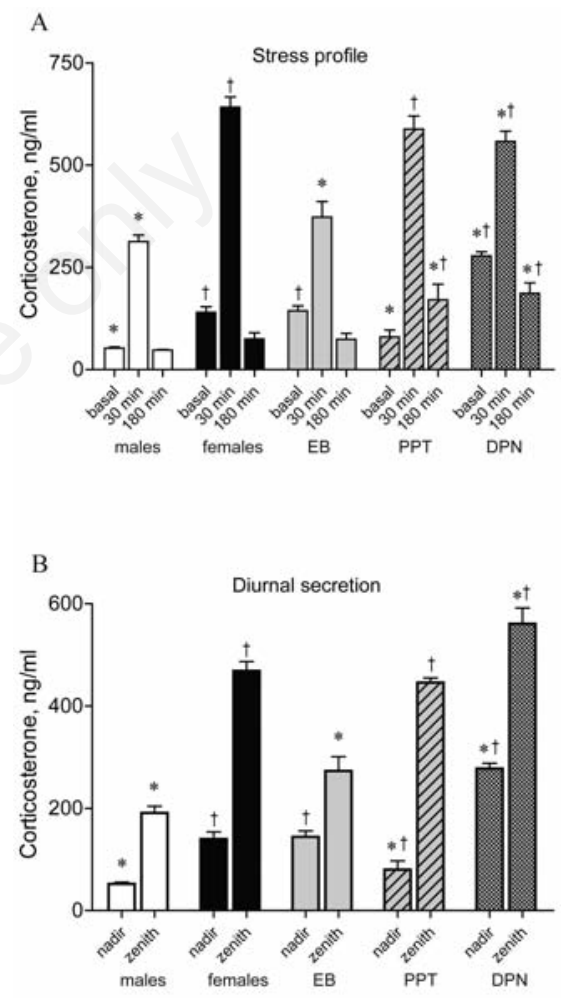

Figure 1. Corticosterone secretory profiles. Neonatal female rats were exposed to estradiol benzoate (EB), PPT (selective ER agonist) or DPN (selective ER agonist) and their basal and stress-induced corticosterone levels assessed during adulthood (PND 100). Comparisons were made with vehicle-treated female and male rats: $A$ ) depicts corticosterone levels at the circadian nadir, and 30 and $180 \mathrm{~min}$ after exposure to a brief emotional stressor; B) diurnal trough and peak levels of corticosterone secretion under quiescent conditions. One-Way-ANOVA were performed for each sampling time point: basal (nadir): $F=62.2 ; 30$ min: $F=26.4 ; 180$ min (Kruskal-Wallis ANOVA) $\mathrm{H}=26.3$; zenith: $\mathrm{F}=45.5$. Bars represent mean \pm SEM of 914 animals/group. Asterisks indicate significant $(P<0.05)$ differences as compared to control vehicle-treated females, crosses indicate significant $(\mathrm{P}<0.05)$ differences vs. control males. 
While this impaired response to the negative feedback actions would be expected to result in increased basal corticosterone levels, PPTtreated females displayed significantly reduced levels of this hormone under basal conditions, as compared to control females (Figure 1B). In marked contrast to the PPT group, DPN-treated females showed high basal levels of corticosterone (Figure 1B) and were unimpaired in the DST (Figure 2A). These differences in feedback efficacy imply differential roles of $E R \alpha$ and ER $\beta$ in the programming and, possibly, regulation of corticosteroid feedback mechanisms.

The hippocampus is implicated as a major site of corticosteroid negative feedback. ${ }^{29}$ It is richly endowed with the two types of corticosteroid receptors, mineralocorticoid (MR) and glucocorticoid (GR) receptors. MR and GR differ in their affinities for corticosterone and together contribute to the maintenance of homeostasis in the HPA axis under basal and stressful conditions. ${ }^{30}$ Analysis of GR and MR mRNA transcripts in the hippocampi of PPTand DPN-treated animals indicated differential regulation of the two receptors by the ER $\alpha$ and ER $\beta$-selective ligands: as compared to vehicle-treated females, PPT- and DPN-treated females showed reduced levels of GR and MR expression, respectively (Figure $2 \mathrm{~B}$ and $2 \mathrm{C}$, $\mathrm{P}<0.0001, \mathrm{~F}=16.6$ and $\mathrm{P}<0.0001, \mathrm{~F}=11.3$ respectively). Together with the above-reported differences in baseline corticosterone secretion and sensitivity to corticosteroid feedback in the PPT- and DPN-treated groups, these observations are consistent with the suggestion that MR are responsible for maintaining HPA axis activity under resting conditions whereas GR are responsible for mediating corticosteroid negative feedback when corticosterone levels exceed a certain threshold. ${ }^{30}$

\section{Opposing behavioral effects of iso-} form-selective neonatal ER activation

The amygdala is another important site of corticosteroid actions. This brain region is not only implicated in the control of emotional behaviors such as anxiety but also of HPA axis activity. However, in contrast to the hippocampus, the amygdala exerts a positive drive on the HPA axis. ${ }^{31}$ Chronically increased levels of corticosterone are closely linked to the expression of anxiety-related behaviors ${ }^{32}$ and, given the results described in the previous section, it was predicted that rats neonatally exposed to DPN would show the highest levels of anxiety. In this study, neonatal administration of PPT and DPN did not significantly influence amygdaloid GR mRNA transcript levels (as compared to vehicle-treated females; Figure 2E). In contrast, amygdaloid MR expression was significantly down- and upregulated after neonatal exposure to PPT and DPN, respectively (Figure $2 \mathrm{D}, \mathrm{P}<0.01$ and $\mathrm{P}<0.0001$ respectively,
$\mathrm{F}=22.2$ ). In the present study, anxiety-related behavior was evaluated in the open field (OF) arena and elevated plus-maze (EPM) by monitoring the following standard parameters, validated to reflect anxiety-related behavior in rodents:21,22 time spent by animal exploring the center area of the unfamiliar $\mathrm{OF}$ and in the open arms of the EPM as well as frequency of

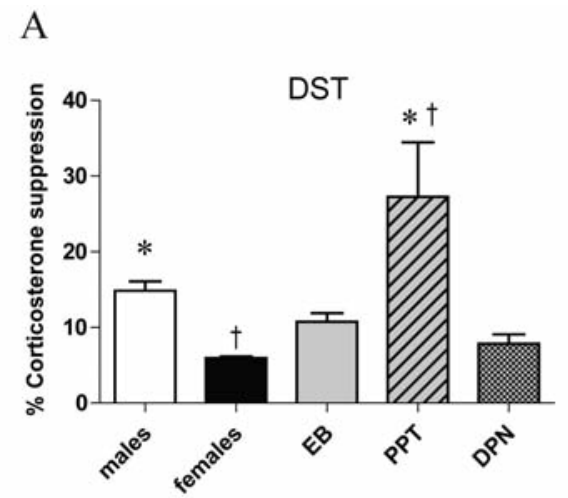

B

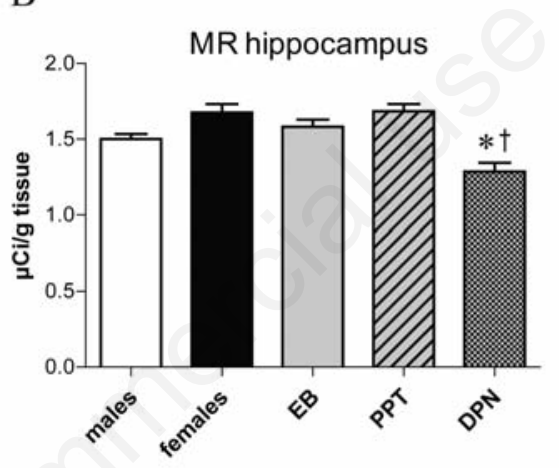

D

C

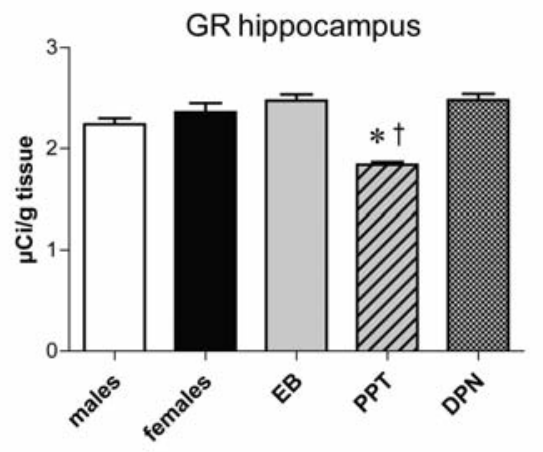

$\mathrm{E}$
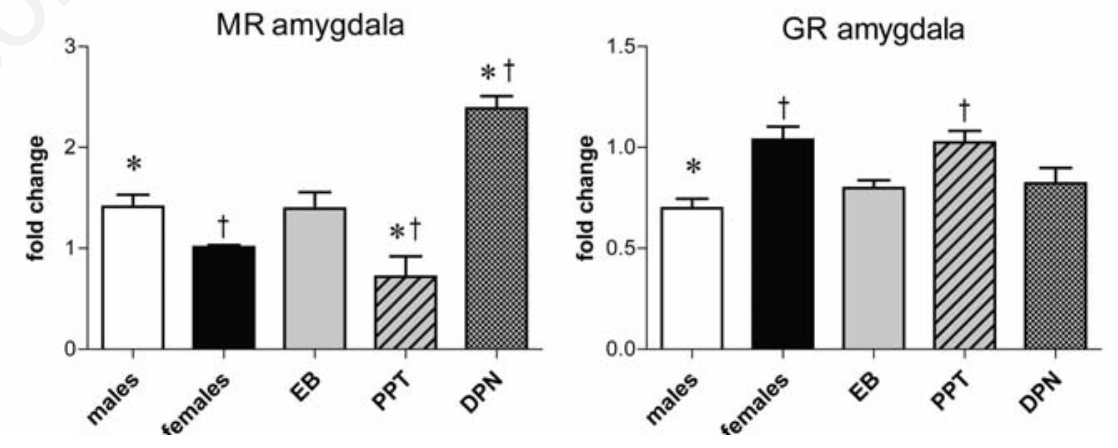

Figure 2. Glucocorticoid negative feedback and its molecular correlates. A) Efficacy of glucocorticoid negative feedback was evaluated by the dexamethasone suppression test (DST). Animals were given $10 \mu \mathrm{g} / \mathrm{kg}$ BW dexamethasone $6 \mathrm{~h}$ before blood samples were collected at the time of the daily peak in corticosterone secretion. Data are presented as a percentage of each individual's peak level of corticosterone secretion on the previous (dexamethasone-free) day. One-Way-ANOVA: $F=10.1$; asterisks indicate $P<0.05$ vs. control females, crosses indicate significant difference vs. control males ( $n=9-14$ animals/group); mRNA expression levels of mineralocorticoid (B, D) and glucocorticoid (C, E) in the hippocampus and amygdala, respectively. mRNA transcripts in the hippocampus were assessed using semi-quantitative in situ hybridization histochemistry. Tissue punches from the amygdala were used to quantify mRNA levels by qPCR; values were normalized against those obtained in control females to yield fold-differences. OneWay-ANOVA: $\mathrm{B}$ ) $\mathrm{F}=11.3$; C) $\mathrm{F}=16.6$; D) $\mathrm{F}=22.2$; $\mathrm{E}$ ) $\mathrm{F}=6.5$. All data are shown as mean \pm SEM of 5-6 animals/group; asterisks indicate significant differences from control females $(P<\mathbf{0 . 0 5})$, crosses indicate significant $(P<\mathbf{0 . 0 5})$ difference vs. control males. 
the vehicle-treated controls on these measures (Figure 3A, 3B and 3C, $\mathrm{P}<0.001$ respectively). These results show that activation of ER $\beta$ in neonatal females results in a hypo-anxious phenotype despite overt hypercorticism. The analysis of the data from the OF test yielded a similar picture: DPN- and vehicle-treated females spent more time exploring the central area of the arena (a sign of reduced anxietyrelated behavior) than males, EB- and PPTtreated animals (Figure $3 \mathrm{D}, \mathrm{P}<0.001, \mathrm{~F}=6.22$ ).

\section{Modulatory influence of ovarian steroids}

Neonatal estrogenization is known to abolish ovarian cyclicity by inducing the so-called interrupted persistent estrus syndrome $e^{33}$ and we previously showed that activation of either ER isoform results in persistent estrus in adulthood. ${ }^{20}$ The above-described mismatches between behavior and endocrine phenotype in the PPT- $v s$ DPN-treated animals led us to consider the potential importance of differential alterations in gonadal status, resulting from isoform-selective neonatal estrogenization, in the observed behavioral phenotypes. The isoform-selectivity of the different neonatal treatments was verified by assessing female sexual behavior. We found that, whereas neonatal treatment with either EB or PPT results in a loss of female sexual behavior, neonatal exposure to DPN does not influence this parameter (data not shown); these results are consistent with our previous findings using other $\mathrm{ER} \alpha$ and ER $\beta$ agonists. ${ }^{20}$ As shown in Figure 4A, estradiol levels in adult females that had been exposed to PPT or DPN during neonatal life were not markedly different from those found in random cycling control females; on the other hand, neonatal EB treatment resulted in significantly reduced levels of estradiol secretion $(\mathrm{P}<0.001, \mathrm{H}=13.13)$. Interestingly, serum progesterone levels were significantly reduced only in the EB and PPT-treated animals $(\mathrm{P}<0.001$ and $\mathrm{P}<0.0001$ respectively, $\mathrm{H}=22.8)$, but not in the DPN-treated group; these findings support the view that neonatal exposure to the ER $\beta$-selective agonist does not abolish the steroid secretory activity of the adult gonad. While ovarian cyclicity (as judged by vaginal epithelial cornification) was abolished by all of the neonatal estrogenization paradigms (data not shown), the degree of ovarian dysfunction, as judged by gonadotropin (LH) secretion (Figure 4C, $\mathrm{P}<0.001, \mathrm{~F}=10.4$ ), ovarian histology (not shown) and ovarian and uterine weights (Figure 4D, $\mathrm{P}<0.0001, \mathrm{~F}=48.6$ and $\mathrm{P}<0.0001, \mathrm{~F}=30.2$ respectively) was graded: EB > PPT > DPN. It is interesting to note that, although elevated HPA axis activity is frequently associated with impaired reproductive function, the DPN-treated animals showed the least degree of ovarian disruption despite their high levels of corticosterone secretion (Figure
1B). Sex differences in basal and stressinduced anxiety are well-known and estrogens have been implicated in the regulation of anxiety in humans and rodents. ${ }^{34}$ Experiments using either pharmacological or genetic approaches have suggested that ER $\beta$ mediate the anxiolytic effects of estrogens. ${ }^{17,35-37}$ The latter, together with the above-reported hypoanxious state of DPN-treated animals prompted us to examine ER $\beta$ expression in the amygdala. As shown in Figure 4E, amygdaloid levels of ER $\beta$ mRNA are sexually differentiated, with females displaying higher ER $\beta$ expression as compared to males $(\mathrm{P}<0.01, \quad \mathrm{~F}=17.3)$. Generally, exposure of neonatal females to $\mathrm{EB}$, PPT or DPN resulted in a significant reduction of ER $\beta$ mRNA levels in the amygdala (Figure $4 \mathrm{E}, \mathrm{P}<0.0001, \mathrm{P}<0.001$ and $\mathrm{P}<0.001$ respectively), but the degree of down-regulation was significantly less in the DPN-treated animals as compared to the EB- and PPT-treated groups $(\mathrm{P}<0.01)$. The latter suggests that neonatally DPN-treated animals are more responsive to estrogens, thus providing an explanation for

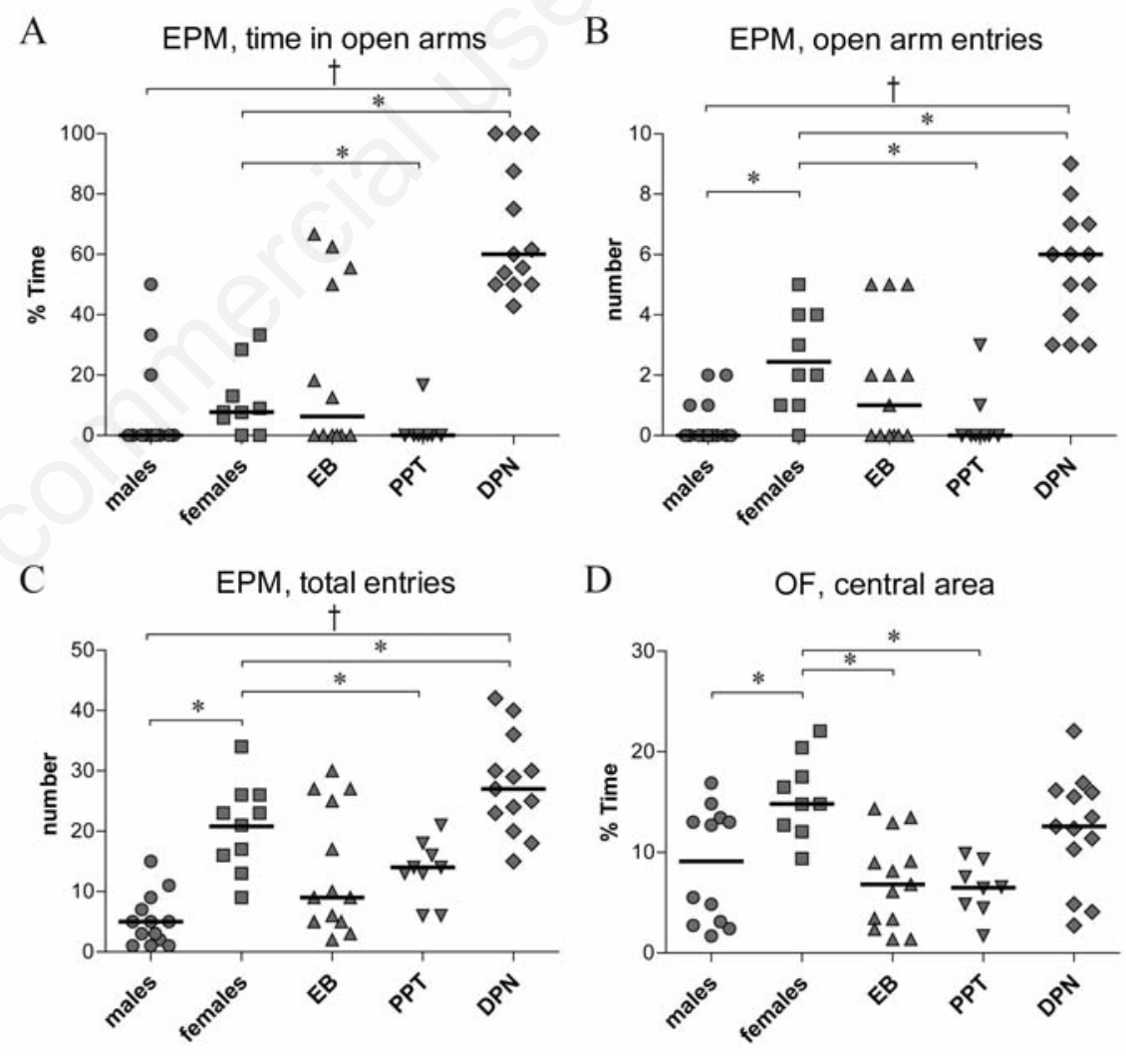

Figure 3. Differential effects on anxiety-related behavior. Measures of anxiety-related behavior were obtained in an open field arena and the elevated plus maze (EPM); animals were monitored in each test over a total of $300 s$ and behaviors were scored by an observer who was blind to the treatments. A) Percentage of time spent in the open arms of the EPM (Kruskal-Wallis-ANOVA H = 29.9); B) Number of entries into the open compartments of the EPM (Kruskal-Wallis-ANOVA $\mathrm{H}=33.4$ ); C) Total number of entries into either open or closed arms of the EPM, serving as an index of locomotor activity (Kruskal-Wallis-ANOVA $\mathrm{H}=31.9$ ); D) Percentage of time spent in the center of an unfamiliar OF (One-Way-ANOVA F = 6.22). The results of each individual are plotted (horizontal lines show group median values), asterisks indicate $\mathrm{P}<0.05$ vs. control females, crosses indicate significant $(\mathrm{P}<0.05)$ difference vs. control males. 
bution.11,12 While ER $\alpha$ are predominantly involved in the regulation of reproductive behavior and hormone secretion as well as growth and maintenance of peripheral reproductive tissues, ER $\beta$ are implicated in the control of a variety of non-reproductive functions, including the regulation of emotion and cognition. ${ }^{17}$

Estradiol can activate both ER $\alpha$ and ER $\beta{ }^{13}$ and current evidence suggests that estrogen actions are determined by cooperative as well as antagonistic actions of the two receptor types. $^{38}$ Previous studies in animals with targeted deletions of ER $\beta$ indicated that this ER isofrom is a crucial mediator of the anxiolytic effects of estrogens. ${ }^{17,35,39}$ In addition, genetic and pharmacological approaches have demonstrated a role for ER $\beta$ in the regulation of corticosterone secretion. On the other hand, mice with $\mathrm{ER} \alpha$ or $\mathrm{ER} \beta$ null mutations do not display clear sexually differentiated HPA axis phenotypes. Accordingly, the goal of this study was to attempt to understand the relative contributions of each ER isoform to the sexual differentiation of the neural substrates responsible for regulation of HPA axis activity and anxiety. Based on the well-established paradigm of neonatal estrogenization of the female rat with $\mathrm{E}_{2}$ - which results in the expression of clear male-like behavioral and neuroendocrine profiles $^{2,9}$ - we here treated neonatal female rats with selective $\mathrm{ER} \alpha$ (PPT) or $\mathrm{ER} \beta$ (DPN) agonists and analyzed their behavioral and endocrine phenotypes during adulthood. The specific features examined included activity of the HPA axis and expression of anxiety-related behavior. Sex differences have been described in both of these functions ${ }^{2,40,41}$ and, in addition, elevated HPA axis activity is positively correlated with increased emotionality and susceptibility to depression and anxiety in humans and animals. ${ }^{1,42}$

As compared to females, males secrete lower amounts of corticosterone under basal conditions and in response to stressful stimuli. ${ }^{2}$ Further, glucocorticoid negative feedback is less efficient in males than in females and thus, shut-off of the HPA axis response to stress is more sluggish in males.,2,428 We previously showed that neonatal administration of $\mathrm{E}_{2}$ defeminizes these measures of HPA axis function in female rats, ${ }^{4}$ a result reproduced in the present work. Our results also show that neonatal activation of either ER $\alpha$ or ER $\beta$ does not defeminize, but clearly disrupts the mechanisms governing HPA axis activity. Interestingly, the two agonists resulted in opposing endocrine phenotypes: whereas animals exposed to neonatal PPT showed femalelike corticosterone secretory response to stress, those exposed to DPN presented with hypersecretion of corticosterone under resting conditions and a relatively blunted endocrine response to stress. Despite these anomalies, the DPN-treated group did not show alterations in their ability to respond to the negative feedback actions of glucocorticoids, as judged by their normal post-stress shut-off of corticosterone secretion and their responses in the DST. In contrast, the animals that had been exposed to neonatal PPT showed marked impairment in terms of glucocorticoid negative feedback.
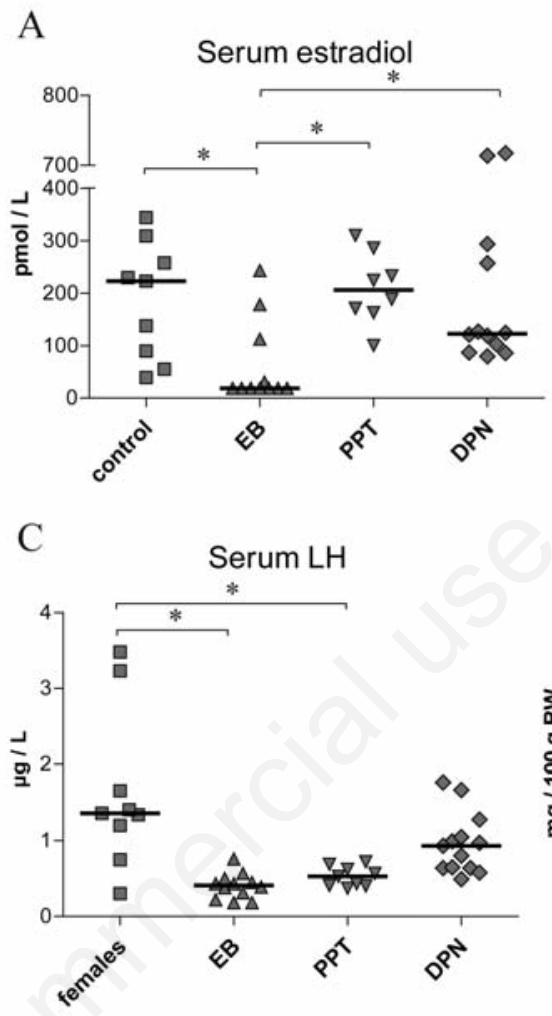

E

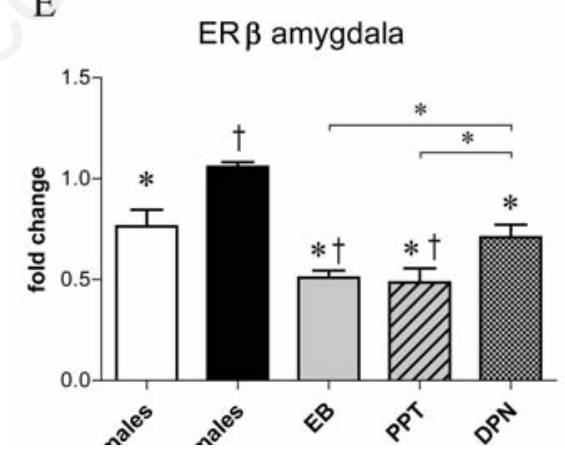

F

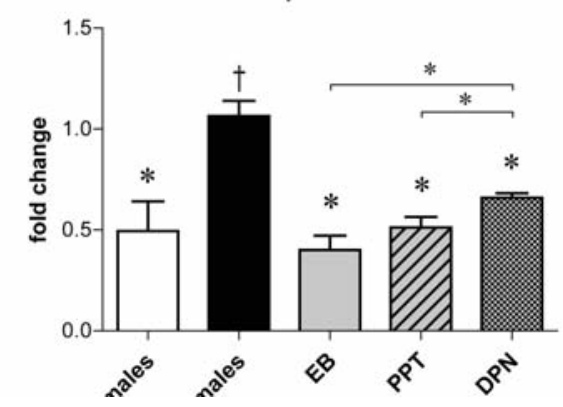

Figure 4. Descriptors of reproductive function and ER $\beta$ expression in the amygdala and hypothalamus. Reproductive parameters evaluated included A) serum estradiol, B) progesterone, C) luteinizing hormone (LH), and D) ovarian and uterine weights. The latter were normalized to body weight (BW) at the time of sacrifice. Individual hormone values and group medians are depicted in $A-C)$. Kruskal-Wallis-ANOVA: $A$ ) $H=13.1$; B) $H$ $=22,8$; C) (One-Way-ANOVA): $\mathrm{F}=111.4$ (asterisks indicate $\mathrm{P}<0.05$ vs. control). Data in D) are means \pm SEM $(n=9-14)$; significant differences from control females are denoted by asterisks $(\mathrm{P}<0.05$; One-Way-ANOVA ovaries: $\mathrm{F}=48.6$; uteri: $\mathrm{F}=30.2)$. Expression levels of ER $\beta$ mRNA in tissue punches from the amygdala $(E)$ and paraventricular nucleus of the hypothalamus (PVN) (F) were obtained by qPCR and values (fold change) are shown normalized against those obtained in control females. Bars represent group means \pm SEM of 5-6 animals per group. One-Way-ANOVA E) $F=17.3$; F) $F=9.3$. Asterisks indicate significant differences vs. control females, crosses indicate significant differences vs. control males $(\mathbf{P}<\mathbf{0 . 0 5})$. 
hypothalamus; within the hypothalamic paraventricular nucleus (PVN), MR and GR are important for inhibiting the central neuropeptidergic (CRH, AVP) drive on the pituitaryadrenal unit. ${ }^{43}$ While MR are suggested to be responsible for maintaining corticosterone levels under basal conditions, GR are implicated in restoring physiological levels of corticosterone secretion following stress. ${ }^{30}$ Given the above-mentioned disruption of HPA axis regulation, it was considered important to gain some insight into the contributory mechanisms by analyzing $M R$ and $G R$ expression in the hippocampus. Our finding that hippocampal MR mRNA expression is reduced in animals given DPN during neonatal development provides an explanation for the elevated basal levels of corticosterone secretion in these animals. Neonatal exposure to PPT resulted in a downregulation of $G R$ expression in the hippocampus, providing a potential mechanistic explanation for the impaired negative feedback efficacy of corticosteroids in the PPTtreated animals.

As already alluded to, chronically elevated levels of corticosterone are frequently associated with a hyperanxious state. ${ }^{32}$ Most studies consider impaired glucocorticoid negative feedback as a factor that contributes to this correlation. ${ }^{44}$ Intriguingly, our assessment of anxiety in animals that had undergone selective neonatal activation of ER $\alpha$ or ER $\beta$ does not support the view that anxiety is a direct correlate of HPA axis activity. On the one hand, we found that neonatal activation of $E R \alpha$ with PPT leads to increased anxiety, an effect that could be explained by the fact that PPT-treated animals are poor responders in the DST and show exaggerated endocrine response to stress; the latter is believed to be a precipitating factor in anxiety disorders. ${ }^{45}$ On the other, we observed reduced anxiety in the DPN-treated rats; these animals showed chronically elevated baseline corticosterone secretion but normal endocrine responses to stress and the DST.

Several groups have suggested a role for GR in the regulation of anxiety. For example, conditional overexpression of $G R$ in the dentate gyrus of the mouse hippocampus reportedly increases anxiety-related behavior as measured in the elevated plus maze, ${ }^{46}$ while $G R$ knockout mice display reduced anxiety-related behaviors. ${ }^{47,48}$ In contrast, forebrain- or amygdala-targeted overexpression of $M R$ is reported to reduce anxiety in rodents. ${ }^{49}$ In this respect, it is notable that animals in which ER $\alpha$ were activated by PPT during neonatal life display significantly reduced levels of amygdaloid MR mRNA as compared to vehicle-treated controls; in contrast, neonatal PPT treatment did not elicit any changes in $G R$ expression in this brain area. Interestingly, the hypo-anxious state observed in DPN-treated rats was associ- ated with a $>2$-fold upregulation of MR expression in the amygdala and we propose that increased MR levels in the amygdala, resulting from neonatal activation of $E R \beta$, serve to reduce anxiety. Amygdaloid MR may also act to buffer against the high levels of corticosterone experienced by animals exposed to the ER $\beta$ agonist during neonatal development by reducing the availability of corticosterone at GR (cf. the MR-GR balance hypothesis proposed by de Kloet and colleagues ${ }^{30}$ ) or the efficacy of GR activity. ${ }^{50,51}$ In addition the increased MR expression in the amygdala of neonatally DPNtreated animals might account for the sluggish acute adrenocritical stress response in these animals (Figure 1A); previous work described a dampening of stress-induced corticosterone secretion in rats overexpressing amygdaloid MR. ${ }^{49}$

Although plausible explanations can be found for the apparently dissociated endocrine and behavioral profiles observed in adult rats whose ER $\alpha$ or ER $\beta$ had been activated during neonatal life, it is important to consider other factors that could have contributed to the development of the specific phenotypes. The neonatal treatments in the present study were used to study the so-called organizational actions of early estrogens on HPA axis function and expression of anxiety. However, results from our previous studies showing that ER $\alpha$ or $\operatorname{ER} \beta$ also differentially organize reproductive development cannot be ignored.20 Here, we found that, whereas neonatal $\mathrm{E}_{2}$ treatment results in hypogonadotropic hypogonadism, anovulatory ovaries and persistent estrus as expected, ${ }^{20,52,53}$ neonatal exposure to PPT and DPN, while also causing persistent cornification of vaginal epithelia, only partially disrupts ovarian activity; in particular, the DPN-treated animals did not differ markedly from vehiclecontrolled rats in this respect and continued to secrete amounts of estrogen that would be sufficient to exert so-called activational actions. Numerous studies have shown that estrogens sex-dependently stimulate the HPA axis in adulthood and there is evidence that low estrogen levels are associated with increased anxiousness in humans and animals.7,34,54 In light of previous studies that described the anxiolytic actions of ER $\beta$ agonists, ${ }^{36}$ the present finding that ER $\beta$ are significantly less downregulated in the amygdala of DPN- treated animals indicates that preserved $\operatorname{ER} \beta$ signaling in the amygdala contributes to the anxiolytic phenotype in the DPN-treated animals. This assumption is further supported by the finding that gonadal secretory activity was not completely abolished in this group, despite the cytological observation of persistent estrus. While levels of estradiol secretion were similar in PPTtreated and control animals, it should be noted that the PPT-treated group showed the highest degree of ER $\beta$ downregulation and high levels of anxiety-related behavior. Accordingly, the distinct behavioral and endocrine phenotypes expressed in adult females that had experienced selective neonatal stimulation of either $\mathrm{ER} \alpha$ or ER $\beta$ likely result from the activational effects of residual estrogen secretion.

Taken together, the present results show that isoform-selective ER activation during neonatal life does not per se contribute to sexspecific organization of the HPA axis, but rather leads to dysregulation of the central mechanisms governing corticosterone secretion under basal and stressful conditions, and dissociate the usual relationship between corticosterone levels and anxiety. In contrast to individual activation of $\mathrm{ER} \alpha$ and $\mathrm{ER} \beta$, dual activation of both ER isoforms with $\mathrm{E}_{2}$ in neonatal females produces a male-like phenotype in which low levels of corticosterone are associated with reduced anxiety-related behavior. Further, our results identify hippocampal and amygdaloid MR and GR expression patterns as correlates of the disrupted endocrine and behavioral profiles. Nevertheless, the molecular and cellular pathways and mechanisms through which neonatal estrogenization exerts its sustained effects on the expression of MR and GR remain to be elucidated. While epigenetic marking of ER-responsive gene loci may account for the sustained effects of neonatal estrogen exposure, 55 the present work indicates that both HPA axis activity and anxiety in neonatally PPT- and DPN-treated animals remain subject to regulation by residual ovarian secretions acting at central ER $\beta$. Since many environmental endocrine disruptors activate $\mathrm{ER} \alpha$ and $\mathrm{ER} \beta \beta^{18,19}$ these findings may be of wider relevance, beyond the present interest in sexual differentiation of the brain and regulation of the endocrine response to stress and stress-related behavior.

Lastly, the present findings have implications for human health since dysregulation of the HPA axis is associated with the pathogenesis of mood and anxiety disorders, both of which show a higher prevalence in women. ${ }^{1}$

\section{References}

1. Young EA. Sex differences and the HPA axis: implications for psychiatric disease. J Gend Specif Med 1998;1:21-7.

2. Patchev VK, Almeida OF. Gender specificity in the neural regulation of the response to stress: new leads from classical paradigms. Mol Neurobiol 1998;16:63-77.

3. Patchev VK, Hayashi S, Orikasa C, Almeida OF. Implications of estrogen-dependent brain organization for gender differences in hypothalamo-pituitary-adrenal regulation. Faseb J 1995;9:419-23.

4. Patchev VK, Hayashi S, Orikasa C, Almeida 
OF. Ontogeny of gender-specific responsiveness to stress and glucocorticoids in the rat and its determination by the neonatal gonadal steroid environment. Stress 1999;3;41-54.

5. Kirschbaum C, Kudielka BM, Gaab J et al. Impact of gender, menstrual cycle phase, and oral contraceptives on the activity of the hypothalamus-pituitary-adrenal axis. Psychosom Med 1999;61:154-62.

6. Atkinson HC, Waddell BJ. Circadian variation in basal plasma corticosterone and adrenocorticotropin in the rat: sexual dimorphism and changes across the estrous cycle. Endocrinology 1997;138:3842-8.

7. Mora S, Dussaubat N, Diaz-Veliz G. Effects of the estrous cycle and ovarian hormones on behavioral indices of anxiety in female rats. Psychoneuroendocrinology 1996;21:609-20.

8. De Nicola AF, Saravia FE, Beauquis J et al. Estrogens and neuroendocrine hypothalamic-pituitary-adrenal axis function. Front Horm Res 2006;35:157-68.

9. Arnold AP, Breedlove SM. Organizational and activational effects of sex steroids on brain and behavior: a reanalysis. Horm Behav 1985;19:469-98.

10. McCormick CM, Furey BF, Child M et al. Neonatal sex hormones have organizational' effects on the hypothalamic-pituitary-adrenal axis of male rats. Brain Res Dev Brain Res 1998;105:295-307.

11. Laflamme N, Nappi RE, Drolet G et al. Expression and neuropeptidergic characterization of estrogen receptors (ERalpha and ERbeta) throughout the rat brain: anatomical evidence of distinct roles of each subtype. J Neurobiol 1998;36:357-78.

12. Shughrue PJ, Lane MV, Merchenthaler I. Comparative distribution of estrogen receptor-alpha and -beta mRNA in the rat central nervous system. J Comp Neurol 1997;388:507-25.

13. Dahlman-Wright K, Cavailles V, Fuqua SA et al. International Union of Pharmacology. LXIV. Estrogen receptors. Pharmacol Rev 2006;58:773-81.

14. Stauffer SR, Coletta CJ, Tedesco R et al. Pyrazole ligands: structure-affinity/activity relationships and estrogen receptor-alphaselective agonists. J Med Chem 2000;43: 4934-47.

15. Meyers MJ, Sun J, Carlson KE, Marriner GA, Katzenellenbogen BS, Katzenellen bogen JA. Estrogen receptor-beta potencyselective ligands: structure-activity relationship studies of diarylpropionitriles and their acetylene and polar analogues. J Med Chem 2001;44:4230-51.

16. Landgraf R, Wigger A, Holsboer F, Neumann ID. Hyper-reactive hypothalamo-pituitary-adrenocortical axis in rats bred for high anxiety-related behaviour. J Neuroendocrinol 1999;11:405-7.

17. Bodo C, Rissman EF. New roles for estrogen receptor beta in behavior and neuroendocrinology. Front Neuroendocrinol 2006;27:217-32.

18. Shanle EK, Xu W. Endocrine disrupting chemicals targeting estrogen receptor signaling: identification and mechanisms of action. Chem Res Toxicol 2011;24:6-19.

19. Swedenborg E, Pongratz I, Gustafsson JA. Endocrine disruptors targeting ERbeta function. Int J Androl 2010;33:288-97.

20. Patchev AV, Gotz F, Rohde W. Differential role of estrogen receptor isoforms in sexspecific brain organization. Faseb J 2004;18:1568-70.

21. Walsh RN, Cummins RA. The Open-Field Test: a critical review. Psychol Bull 1976;83:482-504.

22. File SE, Lippa AS, Beer B, Lippa MT. Animal tests of anxiety. Curr Protoc Neurosci 2004; Chapter 8:Unit 8.3.

23. Patchev VK, Patchev AV. Experimental models of stress. Dialogues Clin Neurosci 2006;8:417-32.

24. Murgatroyd C, Patchev AV, Wu Y et al. Dynamic DNA methylation programs persistent adverse effects of early-life stress. Nat Neurosci 2009;12:1559-66.

25. Miesfeld R, Rusconi S, Godowski PJ et al. Genetic complementation of a glucocorticoid receptor deficiency by expression of cloned receptor cDNA. Cell 1986;46:389-99.

26. Arriza JL, Weinberger C, Cerelli G et al. Cloning of human mineralocorticoid receptor complementary DNA: structural and functional kinship with the glucocorticoid receptor. Science 1987;237:268-75.

27. Whitfield HJ Jr, Brady LS, Smith MA et al. Optimization of cRNA probe in situ hybridization methodology for localization of glucocorticoid receptor mRNA in rat brain: a detailed protocol. Cell Mol Neurobiol 1990;10:145-57.

28. Almeida OF, Canoine V, Ali $\mathrm{S}$ et al. Activational effects of gonadal steroids on hypothalamo-pituitary-adrenal regulation in the rat disclosed by response to dexamethasone suppression. J Neuroendocrinol 1997;9:129-34.

29. van Haarst AD, Oitzl MS, de Kloet ER. Facilitation of feedback inhibition through blockade of glucocorticoid receptors in the hippocampus. Neurochem Res 1997;22: 1323-8.

30. De Kloet ER, Vreugdenhil E, Oitzl MS, Joels M. Brain corticosteroid receptor balance in health and disease. Endocr Rev 1998;19:269-301.

31. Herman JP, Prewitt CM, Cullinan WE. Neuronal circuit regulation of the hypothalamo-pituitary-adrenocortical stress axis. Crit Rev Neurobiol 1996;10:371-94.
32. Pego JM, Sousa JC, Almeida OF, Sousa N. Stress and the neuroendocrinology of anxiety disorders. Curr Top Behav Neurosci 2010;2:97-117.

33. Singh KB. Persistent estrus rat models of polycystic ovary disease: an update. Fertil Steril 2005;84 Suppl 2:1228-34.

34. Frye CA. Steroids, reproductive endocrine function, and affect. A review. Minerva Ginecol 2009;61:541-62.

35. Weiser MJ, Foradori CD, Handa RJ. Estrogen receptor beta in the brain: from form to function. Brain Res Rev 2008;57:309-20.

36. Weiser MJ, Foradori CD, Handa RJ. Estrogen receptor beta activation prevents glucocorticoid receptor-dependent effects of the central nucleus of the amygdala on behavior and neuroendocrine function. Brain Res 2010;1336:78-88.

37. Tomihara K, Soga T, Nomura M et al. Effect of ER-beta gene disruption on estrogenic regulation of anxiety in female mice. Physiol Behav 2009;96:300-6.

38. Gonzales KL, Tetel MJ, Wagner CK. Estrogen receptor (ER) beta modulates ERalpha responses to estrogens in the developing rat ventromedial nucleus of the hypothalamus. Endocrinology 2008;149: 4615-21.

39. Walf AA, Koonce CJ, Frye CA. Estradiol or diarylpropionitrile decrease anxiety-like behavior of wildtype, but not estrogen receptor beta knockout, mice. Behav Neurosci 2008;122:974-81.

40. Johnston AL, File SE. Sex differences in animal tests of anxiety. Physiol Behav 1991;49:245-50.

41. Mitev YA, Darwish M, Wolf SS et al. Gender differences in the regulation of 3 alphahydroxysteroid dehydrogenase in rat brain and sensitivity to neurosteroid-mediated stress protection. Neuroscience 2003;120: 541-9.

42. Martin EI, Ressler KJ, Binder E, Nemeroff CB. The neurobiology of anxiety disorders: brain imaging, genetics, and psychoneuroendocrinology. Clin Lab Med 2010;30: 865-91.

43. Han F, Ozawa H, Matsuda KI et al. Changes in the expression of corticotrophin-releasing hormone, mineralocorticoid receptor and glucocorticoid receptor mRNAs in the hypothalamic paraventricular nucleus induced by fornix transection and adrenalectomy. J Neuroendocrinol 2007;19:22938.

44. De Kloet ER, Vreugdenhil E, Oitzl MS, Joels M. Glucocorticoid feedback resistance. Trends Endocrinol Metab 1997;8:2633.

45. Charmandari E, Tsigos C, Chrousos G. Endocrinology of the stress response. Annu Rev Physiol 2005;67:259-84. 
46. Sarrazin N, Di Blasi F, Roullot-Lacarriere V et al. Transcriptional effects of glucocorticoid receptors in the dentate gyrus increase anxiety-related behaviors. PLoS One 2009;4:e7704.

47. Tronche F, Kellendonk C, Kretz 0 et al. Disruption of the glucocorticoid receptor gene in the nervous system results in reduced anxiety. Nat Genet 1999;23:99103.

48. Boyle MP, Kolber BJ, Vogt SK et al. Forebrain glucocorticoid receptors modulate anxiety-associated locomotor activation and adrenal responsiveness. J Neurosci 2006;26:1971-8.

49. Mitra R, Ferguson D, Sapolsky RM.
Mineralocorticoid receptor overexpression in basolateral amygdala reduces corticosterone secretion and anxiety. Biol Psychiatry 2009;66:686-90.

50. Trapp T, Rupprecht R, Castren M et al. Heterodimerization between mineralocorticoid and glucocorticoid receptor: a new principle of glucocorticoid action in the CNS. Neuron 1994;13:1457-62.

51. Liu W, Wang J, Sauter NK, Pearce D. Steroid receptor heterodimerization demonstrated in vitro and in vivo. Proc Natl Acad Sci USA 1995;92:12480-4.

52. Ikeda Y, Nagai A, Ikeda MA, Hayashi S. Neonatal estrogen exposure inhibits steroidogenesis in the developing rat ovary. Dev Dyn 2001;221:443-53.

53. Nakamura T, Katsu Y, Watanabe H, Iguchi T. Estrogen receptor subtypes selectively mediate female mouse reproductive abnormalities induced by neonatal exposure to estrogenic chemicals. Toxicology 2008;253:117-24.

54. Viau V, Meaney MJ. Variations in the hypothalamic-pituitary-adrenal response to stress during the estrous cycle in the rat. Endocrinology 1991;129:2503-11.

55. Nugent BM, McCarthy MM. Epigenetic underpinnings of developmental sex differences in the brain. Neuroendocrinology 2011;93:150-8. 